02

\title{
Спектрометрический метод определения несвязанного йода в йодированных эфирах жирных кислот
}

\author{
(C) В.В. Клюбин ${ }^{1}$, К.А. Клюбина ${ }^{2}$, К.Н. Маковецкая ${ }^{1}$ \\ ${ }^{1}$ Российский научный центр радиологии и хирургических технологий, \\ 197758 Санкт-Петербург, Россия \\ ${ }^{2}$ Государственный морской технический университет, \\ 190008 Санкт-Петербург, Россия \\ e-mail: kljubin@mail.ru
}

Поступила в редакцию 06.06.2017 г.

В окончательной редакции 20.08.2017 г.

Показана возможность измерения концентрации свободного йода в образцах йодированных эфиров жирных кислот с использованием электронных спектров поглощения их растворов в этиловом спирте. Предложенный метод достаточно прост в реализации и обладает высокой чувствительностью, с помощью этого метода можно обнаружить присутствие менее 10 ppm несвязанного йода в йодированных препаратах. На примере рентгеноконтрастного препарата Липиодола показано, что с помощью этого метода легко регистрируются небольшие количества свободного йода в образцах, содержание связанного йода в которых доходит до 40 wt.\%.

DOI: $10.21883 /$ OS.2018.01.45357.135-17

\section{Введение}

В последние годы в медицине активно используют полимерные материалы, содержащие йод. Процесс синтеза йодсодержащих препаратов включает в себя стадию присоединения йода по двойным связям непредельных жирных кислот. Неприсоединившийся йод удаляется из препарата специальными методами очистки. Однако часть свободного йода может остаться в веществе, что недопустимо, так как присутствие даже небольшого количества молекулярного йода в лекарственном препарате может привести к губительным последствиям для человека. В связи с этим необходимо тщательно контролировать процесс очистки синтезируемого вещества от свободного йода. Кроме того, йодсодержащие препараты на практике оказываются неустойчивыми. В процессе хранения из них может выделяться йод, что снижает качество этих веществ и делает их непригодными для дальнейшего использования. Поэтому необходимо контролировать содержание свободного йода и в процессе хранения йодсодержащих препаратов.

В настоящее время существует большое число методов, позволяющих определять присутствие йода в различных веществах [1-8], но все они либо трудоемки, либо требуют сложного оборудования. В настоящей работе для количественного определения содержания свободного йода в йодсодержащих веществах предлагается простой и достаточно точный метод, основанный на измерении оптической плотности растворов испытуемых веществ на одной длине волны $360 \mathrm{~nm}$. Этот метод является весьма перспективным, так как в этой области спектра растворы йода имеют достаточно большое сечение поглощения. Настоящая работа является продолжением цикла исследований [9], в которых показана перспективность данного способа обнаружения даже небольших количеств молекулярного йода.

\section{Экспериментальная часть}

В качестве объекта исследований в работе использовались образцы йодированных эфиров жирных кислот. В своем составе они могут содержать до $40 \%$ связанного йода, что делает их очень полезными для контрастирования различных органов человеческого организма при проведении рентгеновских исследований. Электронные спектры эфиров жирных кислот располагаются в ультрафиолетовой области. Спектр поглощения этилового эфира олеиновой кислоты (ЭЭОК) показан на рис. 1 (кривая 1). Спектр йодированного продукта представлен

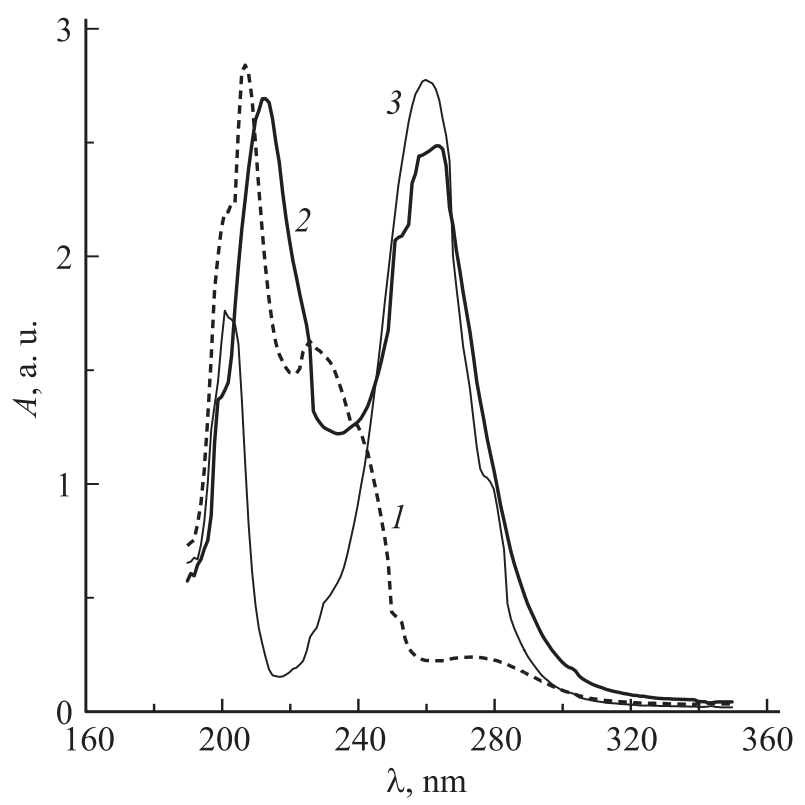

Рис. 1. Спектры поглощения растворов ЭЭОК (1), йодированного ЭЭОК (2) и йодистого этила (3) в этиловом спирте. 
на этом же рисунке кривой 2. Йодированный полимер получается в результате присоединения йодистого водорода НІ к этиловому эфиру олеиновой кислоты [10]. Спектры рис. 1 были измерены в очень разбавленных растворах с помощью спектрометра ПЭ5400УФ (производство ЭКОХИМ, Санкт-Петербург). Из спектров рис. 1 следует, что в йодированном полимере возникает дополнительная полоса поглощения с максимумом на длине волны $264 \mathrm{~nm}$. Эта полоса появляется из-за того, что в углеводородной цепи возникают $\mathrm{C}-\mathrm{I}$-связи. Такая же полоса присутствует в спектрах поглощения растворов йодистого метила $\mathrm{CH}_{3} \mathrm{I}$ и этила $\mathrm{C}_{2} \mathrm{H}_{5} \mathrm{I}$. На рис. 1 для сравнения приведен спектр поглощения раствора $\mathrm{C}_{2} \mathrm{H}_{5} \mathrm{I}$ в этаноле (кривая 3). Он также содержит полосу с максимумом на длине волны $260 \mathrm{~nm}$, характерную для связи $\mathrm{C}-\mathrm{I}$. Таким образом, полоса поглощения с максимумом на длине волны $264 \mathrm{~nm}$ в спектрах йодированного ЭЭОК говорит о наличии связанного йода в молекулах этого вещества.

Йодированный ЭЭОК имеет слабую желтоватую окраску и хранится достаточно долго в темноте и в инертной среде (например, в откаченной запаянной пробирке или в аргоне). На свету и при контакте с кислородом воздуха происходит разрушение этого продукта, он темнеет, становится коричневым, а после длительного контакта с воздухом и светом становится темно-коричневым с черным оттенком. Проведя исследования спектров поглощения растворов, приготовленных из вещества, подвергнутого длительному хранению в незапаянном сосуде на свету, мы заметили, что в нем выделяется свободный йод, что и приводит к изменению его цвета.

На рис. 2 показаны электронные спектры поглощения растворов йодированного ЭЭОК: свежеприготовленного (кривые 1 и 2) и после месячного хранения на свету в незапаянном сосуде при комнатной температуре (кривая 3). Кривая 1 показывает электронный спектр поглощения йодированного ЭЭОК, полученный при сильном разбавлении в этиловом спирте (разбавлен в 200 раз). В этом спектре присутствует полоса поглощения связанного йода с максимумом на длине волны $264 \mathrm{~nm}$. При меньшем разбавлении (в 40 раз) коротковолновые полосы в спектре не разрешаются, а наблюдается одна широкая полоса в области от 190 до $320 \mathrm{~nm}$ (кривая 2). В области более длинных волн от 320 до $420 \mathrm{~nm}$ оптическая плотность этого раствора монотонно уменьшается, что говорит о том, что в этой области у йодированного эфира нет характерного поглощения. Иная картина наблюдается в спектре образца, выдержанного на свету в течение длительного времени (кривая 3). В спектре этого раствора в области $320-420 \mathrm{~nm}$ возникает дополнительная полоса поглощения с максимумом на длине волны $360 \mathrm{~nm}$. Интенсивность этой полосы для слабо окрашенных образцов небольшая, но увеличивается по мере того, как они становятся более темными.

Как показали наши исследования спектров растворов молекулярного йода и его различных соединений $[11,12]$,

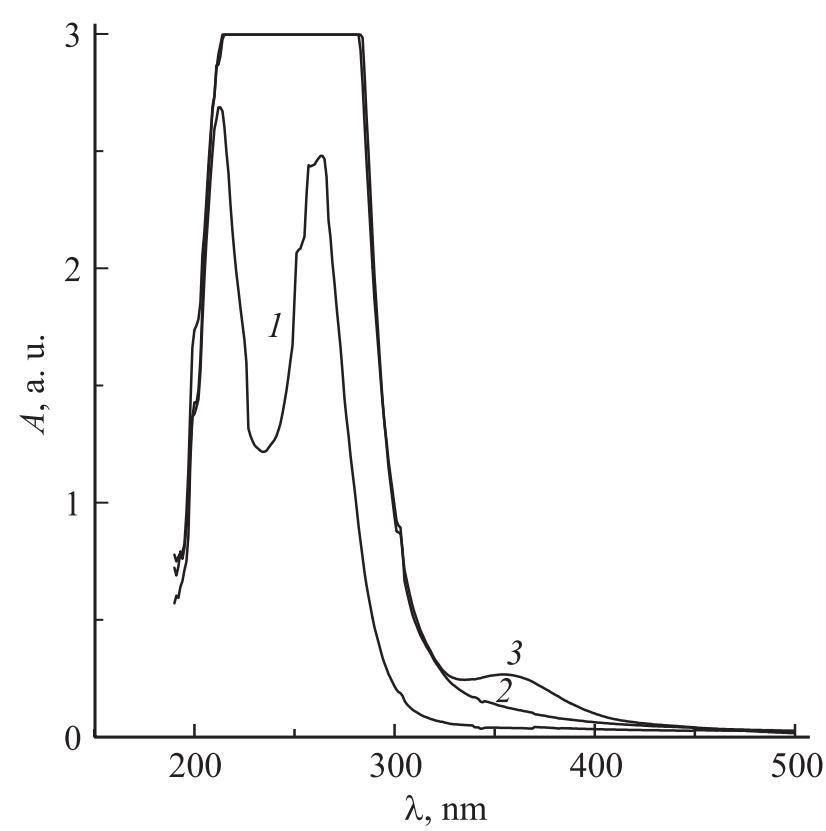

Рис. 2. Спектры поглощения растворов в этиловом спирте: 1 - свежеприготовленного йодированного ЭЭОК конц. $0.5 \mathrm{wt} . \%, 2$ - свежеприготовленного йодированного ЭЭОК конц. 2.5 wt.\%, 3 - йодированного ЭЭОК после месячного хранения конц. $2.5 \mathrm{wt} . \%$.

дополнительная полоса с максимумом на длине волны $360 \mathrm{~nm}$ связана с появлением в растворе аниона трийодида $\mathrm{I}_{3}^{-}$. В образцах йодированных ЭЭОК в процессе длительного хранения происходит отщепление йода, который в разбавленных растворах превращается в сложный анион $\mathrm{I}_{3}^{-}$. Возникновение дополнительной полосы поглощения с максимумом на длине волны $360 \mathrm{~nm}$ в образцах йодированных ЭЭОК позволило нам разработать удобный и достаточно чувствительный метод контроля содержания свободного йода в них. Суть метода состоит в том, что для получения информации о наличии свободного йода необходимо измерять оптическую плотность разбавленных растворов тестируемых образцов на одной длине волны $360 \mathrm{~nm}$. В качестве растворителя в этих измерениях следует брать этиловый спирт. Этанол является хорошим растворителем для молекулярного йода, но он плохо растворяет йодированную ЭЭОК. При этом происходит хорошее разделение свободного йода от связанного.

Предлагаемый метод хорошо работает в достаточно широкой области концентраций свободного йода, где оптическая плотность растворов $A$ на длине волны $360 \mathrm{~nm}$ линейным образом зависит от концентрации $C$ растворенного вещества.

$$
A=\varepsilon C l \text {. }
$$

Для проверки зависимости (1) было приготовлено несколько растворов йода в этаноле с различной концентрацией. Измерения оптической плотности всех приготовленных образцов проводились в кварцевой кюве- 
те толщиной $l=5 \mathrm{~mm}$. По усредненным данным был построен график зависимости оптической плотности $A$ растворов йода на длине волны $360 \mathrm{~nm}$ от концентрации йода $C$. Этот график приведен на рис. 3 . Видно, что экспериментальные точки в области исследуемых концентраций хорошо укладываются на линейную зависимость. Экспериментальные данные обрабатывались по методу наименьших квадратов, значение тангенса угла наклона полученной прямой мы использовали для вычисления коэффициента поглощения исследуемых растворов. Многократные измерения показали, что коэффициент поглощения растворов йода в этаноле на длине волны $360 \mathrm{~nm}$ равняется

$$
\varepsilon=0.0036 \pm 0.0001 \frac{1}{\mathrm{~mm} \times \mathrm{ppm}} .
$$

При вычислении коэффициента поглощения $\varepsilon$ мы брали значение концентрации йода в ppm, а толщину измерительной кюветы в $\mathrm{mm}$.

В процессе разработки предлагаемого метода определения концентрации свободного йода в йодированных образцах жирных кислот мы установили, что при вычислениях необходимо учитывать влияние основной полосы поглощения молекул йодированного полимера на поглощение анионов $\mathrm{I}_{3}^{-}$. На рис 2 видно, что полоса поглощения аниона $\mathrm{I}_{3}^{-}$(кривая 3) возникает на фоне подставки, представляющей собой „хвост“ основной полосы поглощения йодированного ЭЭОК (кривая 2). Учет влияния этой „подставки“ можно выполнить, измерив значение оптической плотности образцов, не содержащих свободный йод, на длине волны $360 \mathrm{~nm}$. Полученные значения поправки $A_{\mathrm{o}}$ приведены в таблице.

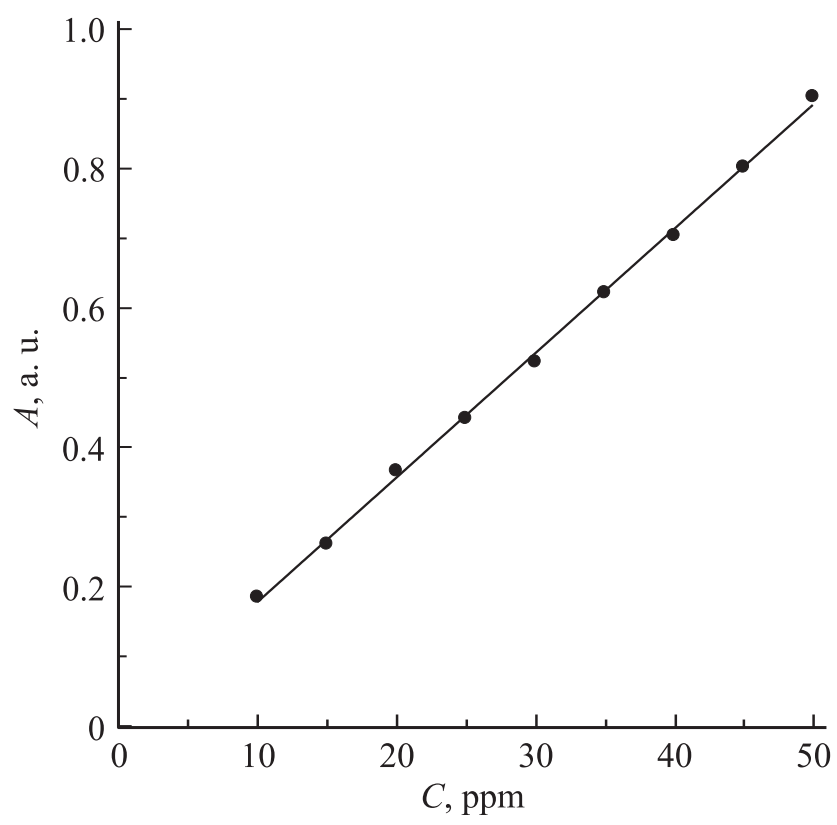

Рис. 3. Зависимость оптической плотности поглощения растворов молекулярного йода на длине волны $360 \mathrm{~nm}$ от концентрации йода в этаноле.
Величина поправки $A_{\text {o }}$ при различных разбавлениях йодированного ЭЭОК

\begin{tabular}{l|c|c|c|c}
\hline Степень разбавления, $N$ & 20 & 30 & 40 & 50 \\
\hline $\begin{array}{l}\text { Оптическая плотность } \\
\text { на } 360 \mathrm{~nm}, A_{0}, \mathrm{~mm}^{-1}\end{array}$ & 0.042 & 0.035 & 0.029 & 0.022
\end{tabular}

Таким образом, для расчета концентрации свободного йода в исследуемых образцах йодированного ЭЭОК необходимо использовать следующую формулу:

$$
C=\frac{N}{\varepsilon}\left(\frac{A_{X}}{l}-A_{\mathrm{o}}\right)=270 N\left(\frac{A_{X}}{l}-A_{\mathrm{o}}\right),
$$

где $A_{X}$ - оптическая плотность исследуемого образца на длине волны $360 \mathrm{~nm}, N-$ степень разбавления исследуемого образца, $l$ - толщина измерительной кюветы в $\mathrm{mm}, A_{\mathrm{o}}$ - поправка, связанная с влиянием основной полосы поглощения йодированного ЭЭОК.

Значение концентрации свободного йода в исследуемых образцах, вычисленное по формуле (2), получается в единицах ppm (1 миллионная часть вещества).

Для нахождения концентрации свободного йода $C$ в тестируемом образце йодированного ЭЭОК необходимо выполнить следующие операции. Анализируемый образец разбавляется в этиловом спирте в 20-50 раз (по массе). Затем измеряется оптическая плотность $A_{X}$ разбавленного раствора на длине волны $\lambda=360 \mathrm{~nm}$. Полученное значение подставляется в формулу (2), из которой получается концентрация свободного йода в исследуемом образце.

Для получения достоверной информации определение концентрации свободного йода необходимо выполнить 3-5 раз, каждый раз заново разбавляя исходный образец (не стоит использовать доли одного и того же раствора, приготовленного в большом количестве). Полученные результаты необходимо усреднить, среднее значение дает искомое содержание йода в исследуемом образце. Измерения необходимо проводить в кварцевых оптических кюветах. Для определения концентрации свободного йода по данной методике требуется около $1 \mathrm{ml}$ препарата.

Предложенную методику мы опробовали на образце рентгеноконтрастного вещества Липиодол. Его выпускает французская фармацевтическая промышленность и он широко используется у нас в стране. Мы взяли свежий образец Липиодола и измерили электронные спектры поглощения его растворов в этаноле. Эти спектры, измеренные при различном разбавлении, показаны на рис. 4 (кривые 1 и 2). Полученные кривые показывают, что в образце Липиодола нет свободного йода. Полоса поглощения с максимумом на длине волны $264 \mathrm{~nm}$, присутствующая в спектре (1), говорит о том, что в полимере есть связанный йод. Затем в Липиодол было добавлено немного раствора молекулярного йода в количестве $0.2 \mathrm{mg}$ на $1 \mathrm{~g}$ препарата $(200 \mathrm{ppm})$. В спектре такого образца появилась полоса, связанная со свободным 


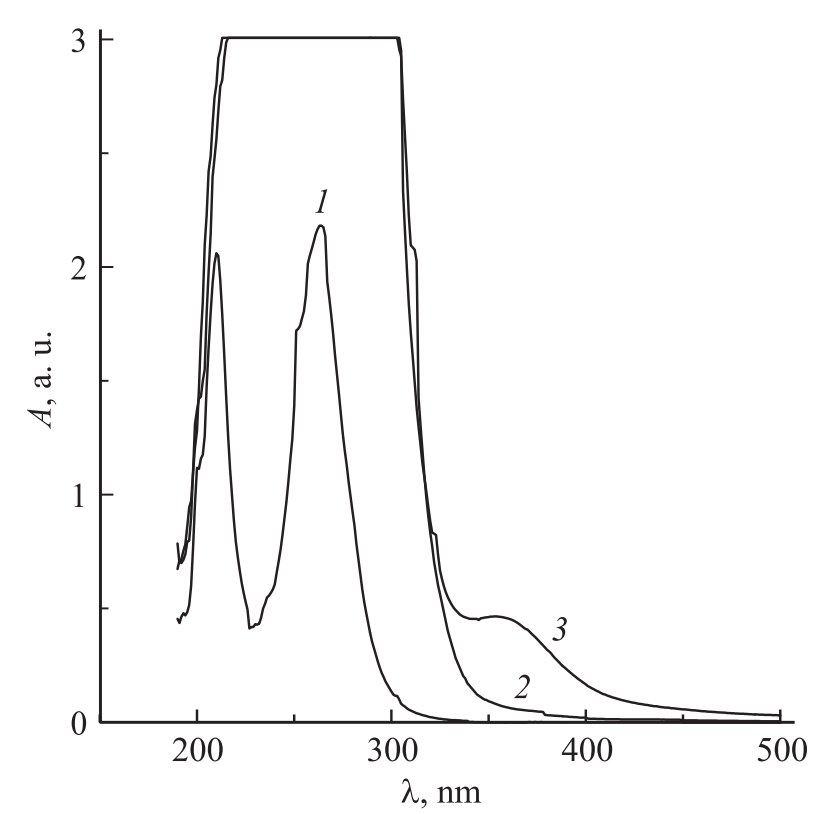

Pис. 4. Спектры поглощения растворов Липиодола в этаноле: 1 - степень разбавления 400, 2 - степень разбавления 20, 3 - в Липиодол добавлено $200 \mathrm{ppm}$ йода, степень разбавления 20.

йодом, это плечо с максимумом на длине волны $360 \mathrm{~nm}$ на кривой 3 рис. 4. Измерив оптическую плотность этого раствора на $360 \mathrm{~nm}$ и воспользовавшись формулой (2), мы получили, что в этом образце действительно содержится свободный йод в количестве 200 ppm.

\section{Заключение}

Предложена методика измерения концентрации несвязанного йода в образцах йодированных эфиров жирных кислот, использующая измерение оптической плотности растворов исследуемых образцов в этаноле на длине волны $360 \mathrm{~nm}$. Методика отличается простотой и высокой чувствительностью. Концентрация свободного йода получается путем расчета с использованием достаточно простой формулы (2) и не требует дополнительных измерений, связанных с построением калибровочных кривых.

\section{Список литературы}

[1] Определение иода в соли поваренной пищевой иодированной. МУК 4.1.699-98. М.: Интерсэн, 1998.

[2] Мохнач В.О., Пропп Л.Н. // Журн. аналит. хим. 1968. Т. 23. B. 2. C. 255

[3] Mitsuhashi T., Kaneda Y. // Assoc. Anal. Chem. 1990. V. 73. N 5. P. 790

[4] Bakker H.J. // J. Assoc. Anal. Chem. 1977. V. 60. N 6. P. 1307.

[5] Maros L., Kaldy M., Igaz S. // Anal. Chem. 1989. V. 61. N 7. P. 733.

[6] Lin F.M., Wu H.L., Kou H.S., Lin S.J. // J. Agric. Food Chem. 2003. V. 51. N 4. P. 867.
[7] Williams S. Official Methods of Analysis of the Association of Official Analytical Chemists. Arlington, 1995. 299 p.

[8] Hu W., Hasebe K., Tanaka K. et al. // Anal. Chem. 1999. V. 71. N 8.P. 1617.

[9] Клюбин В.В., Клюбина К.А., Маковецкая К.Н. // Опт. и спектр. 2016. Т. 120. № 4. С. 46.

[10] Гранов А.М., Маковецкал К.Н., Карелин М.И., Гранов Д.А., Таразов П.Г. Патент РФ № 2448732, 2012.

[11] Клюбин В.В., Клюбина К.А., Маковецкая К.Н. // Журн. физ. химии. 2017. Т. 91. № 4. С. 620.

[12] Клюбин В.В., Клюбина К.А., Маковецкая К.Н. // Журн. физ. химии. 2017. Т. 91. № 6. С. 